\title{
THE FEBRUARY MEETING IN CHICAGO
}

The four hundred thirty-third meeting of the American Mathematical Society was held at the University of Chicago, Chicago, Illinois, on Saturday, February 28, 1948, with a total attendance of one hundred seventeen, including the following one hundred ten members.

E. J. Akutowicz, A. A. Albert, W. R. Allen, Reinhold Baer, J. H. Bell, Herman Betz, S. F. Bibb, R. H. Bing, D. G. Bourgin, H. R. Brahana, G. W. Brown, R. H. Bruck, P. B. Burcham, Lee Byrne, K. H. Carlson, R. E. Carr, E. D. Cashwell, T. E. Caywood, E. W. Chittenden, A. M. Christiansen, H. J. Cohen, J. J. Corliss, J. B. Crabtree, H. J. Curtis, W. M. Davis, D. M. DeWitt, Flora Dinkines, John DyerBennet, J. H. Engel, G. M. Ewing, J. V. Finch, Harley Flanders, R. S. Fouch, J. S. Frame, Evelyn Frank, C. G. Fry, R. E. Fullerton, Albert Furman, H. H. Goldstine, S. H. Gould, Charles Hatfield, I. R. Hershner, Fritz Herzog, Edwin Hewitt, E. H. C. Hildebrandt, J. D. Hill, D. L. Holl, C. C. Hsiung, H. K. Hughes, T. J. Jaramillo, Meyer Jerison, Madeline Johnsen, W. C. Kalinowski, Irving Kaplansky, William Karush, Leo Katz, L. M. Kelly, M. Z. Krzywoblocki, E. P. Lane, W. G. Leavitt, Walter Leighton, M. M. Lemme, Benjamin Lepson, C. T. Loo, W. J. Lyche, A. W. McGaughey, Morris Marden, M. S. Matchett, H. F. Mathis, C. W. Moran, E. J. Moulton, S. B. Myers, E. P. Northrop, F. S. Nowlan, E. N. Oberg, E. H. Ostrow, Gordon Pall, P. M. Pepper, Everett Pitcher, J. C. Polley, D. H. Potts, A. L. Putnam, O. W. Rechard, W. T. Reid, Haim Reingold, L. D. Rodabaugh, G. F. Rose, Arthur Rosenthal, C. H. Rust, H. J. Ryser, J. M. Sachs, R. G. Sanger, L. J. Savage, A, C. Schaeffer, W. T. Scott, I. E. Segal, Seymour Sherman, M. F. Smiley, A. H. Smith, R. H. Stark, M. P. Steele, H. E. Stelson, B. M. Stewart, M. H. Stone, D. G. Velesz, C. P. Wells, L. R. Wilcox, C. O. Williamson, J. L. Zemmer, Antoni Zygmund.

At 9:00 A.M., with Professor A. A. Albert presiding, Professor André Weil delivered an address entitled, Abelian varieties. A session for short research papers was held at 10:15 A.M. with Professor Gordon Pall presiding. The meeting was closed at 12:30 P.M.

In the interval between the two sessions the Associate Secretary read a letter from Professor Inzinger, President of the Austrian Mathematical Society, asking for names of mathematicians who would be interested in receiving free a copy of the Nachrichten der Mathematischen Gesellschaft in Wein.

Local arrangements for this meeting were in charge of Dr. J. B. Crabtree.

There follow the complete abstracts of the papers presented at this meeting. The papers presented by title are designated by the letter "t." Paper no. 216 was presented by Professor Hatfield.

\section{Algebra and Theory of Numbers}

214t. Marshall Hall: Subgroups of finite index in free groups. 
A standard representation for subgroups $U$ of free groups is given in a paper (M. Hall and T. Rad6, On Schreier systems in free groups, Bull. Amer. Math. Soc. Abstract 53-11-357) to appear in the Transactions of the American Mathematical Society. Using this representation, some new relationships are established for subgroups of finite index. In particular, the total length of a system of generators for $U$ is given in terms of the total length of coset representatives. Also a formula is found giving the number of distinct subgroups of index $n$ in the free group with $r$ generators. (Received January 22, 1948.)

\section{5t. Sam Perlis and Gordon Walker: $A$ generalization and new proof of a theorem on scalar extensions of a field.}

The following theorem is established. Let $K(\phi)$ be a separable finite extension of the field $K$, and $F$ be any scalar extension of $K$. Let the minimum function $f(x)$ of $\phi$ over $K$ have the irreducible factorization $f(x)=\prod_{i=1}^{r} f_{i}(x)$ in the polynomial domain $F[x]$; then regarding $K(\phi)$ as an algebra over $K$, its scalar extension $K(\phi)_{F}$ has the structure $K(\phi)_{F}=F\left(\phi_{1}\right) \oplus \cdots \oplus F\left(\phi_{r}\right)$ where $f_{i}\left(\phi_{i}\right)=0$ and $F\left(\phi_{i}\right)$ is the stem field of $f_{i}(x)$. When $F$ is (or contains) the scalar root field over $K$ of $K(\phi)$, each $f_{i}(x)$ has degree one and a well known theorem results (A. A. Albert, Structure of algebras, Amer. Math. Soc. Colloquium Publications, vol. 24, Theorem 26). The proof, which consists of showing that the minimum function of $\phi_{1}+\cdots+\phi_{r}$ is $f(x)$, is simpler than known proofs of the corollary. (Received January 20,1948.)

\section{ANALYsis \\ 216. R. H. Cameron and Charles Hatfield: On the summability of certain orthogonal developments of nonlinear functionals.}

The authors show that the Fourier-Hermite expansion (Ann. of Math. vol. 48 (1947) pp. 385-392) of a bounded Wiener measurable (nonlinear or linear) functional is Abel summable (in an appropriate infinite-dimensional sense) to the value of the functional at each point where the functional is continuous in the Hilbert topology. The Fourier-Hermite set of orthogonal functionals is a set of finite products of Hermite polynomials of Stieltjes integrals $\int_{0}^{1} \alpha_{j}(t) d x(t)$ in which $x$ is the variable function and the $\alpha_{j}(t)$ form a C. O. N. on $0 \leqq t \leqq 1$. In the present case, the authors have taken $\alpha_{j}(t)$ to be $2^{1 / 2} \cos (j-1 / 2) \pi t$. (Received January 19, 1948.)

\section{Evelyn Frank: Orthogonality properties of C-fractions. II.}

This paper constitutes an extension to the general case of special orthogonality relations for $\alpha$-regular and regular $C$-fractions previously considered by the author (Bull. Amer. Math. Soc. Abstract 54-3-132). Consider the polynomials $B_{p}^{*}(z)$ $=z^{n_{p}} B_{p}(1 / z), p=0,1, \cdots$. Here $n_{p}=s_{p}$ if $s_{p} \geqq t_{p}$, or $n_{p}=t_{p}$ if $s_{p}<t_{p} ; s_{p}$ is the degree of the numerator and $t_{p}$ the degree of the denominator $B_{p}(z)$ of the $p$ th approximant of any $C$-fraction $1+a_{1} z^{\alpha_{1}} / 1+a_{2} z^{\alpha_{2}} / 1+\cdots$ (cf. Frank, Amer. J. Math. vol. 68 (1946)). Let the sequence $\left\{c_{p}\right\}$ be the coefficients of the power series corresponding to the $C$-fraction, and let $S$ be defined as the operator which replaces every $z^{p}$ by $c_{p+1}$ in any polynomial upon which it operates. Then the orthogonality relations $S\left(B_{p}^{*}(z) B_{q}^{*}(z)\right)=0$ if $n_{q}=0,1, \cdots, \quad \sum_{i=1}^{p+1} \alpha_{i}-n_{p}-2, S\left(B_{p}^{*}(z) B_{q}^{*}(z)\right)=(-1)^{p} a_{1} a_{2}$ $\cdots a_{p+1}$ if $n_{q}=\sum_{i=1}^{p+1} \alpha_{i}-n_{p}-1$ hold relative to $S$. A necessary condition for the existence of the polynomials $B_{p}^{*}(z)$ is the vanishing of certain determinants $\Delta$ with elements $c_{p}$ (op. cit. p. 93), and the nonvanishing of certain others. Conversely, from 
the $c_{p}$ and these determiant conditions, there may be determined uniquely the polynomials $B_{p}^{*}(z)$ such that the above orthogonality relations hold. (Received January 21, 1948.)

\section{Morris Marden: On the zeros of certain real rational and meromorphic functions.}

The Jensen-Walsh theorems on the zeros of the derivative of a real polynomial are generalized to real rational functions of the form $F(z)=\sum_{j=1}^{n} \rho_{j} e^{i \omega_{j}} /\left(z-c_{j}\right)$ with $\left|\omega_{j}\right|<\pi / 2$ in this paper. By $K_{j}$ is denoted the circle passing through the points $c_{j}$ and $\bar{c}_{j}$ and having its center at a point $k_{j}$ such that angle $\bar{c}_{j}, c_{j}, k_{j}$ measured counterclockwise is $\omega_{j}$. It is then proved that all the real zeros of $F(z)$ lie in the interval containing both all the real $c_{j}$ and all the $k_{j}$, that between any two real $c_{j}$ lie an odd number of zeros of $F(z)$ and that on any interval of the real axis containing no $c_{j}$ there lies at most one real zero of $F(z)$. It is also proved that no non-real zero of $F(z)$ may lie outside of all the circles $K_{j}$ and that, if $n_{1} \leqq n$ points $c_{j}$ lie in a region $R$ consisting of the closed interiors of some $K_{j}$ but not overlapping the closed interiors of the remaining $K_{j}$, then at least $n_{1}-1$ and at most $n_{1}+1$ zeros of $F(z)$ lie in $R$. By iteration these theorems applied to the sequence of real rational functions $f_{p+1}(z) / f_{p}(z)=F_{p}(z)$

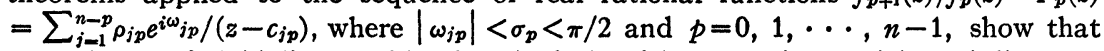
no real zero of $F_{p}(z)$ lies outside of each circle with center $\left(c_{j 0}+\bar{c}_{j 0}\right) / 2$ and diameter $\left|c_{j 0}-\bar{c}_{j 0}\right|\left(\cos \sigma_{0}\right) \prod_{j=0}^{p} \csc \sigma_{j}$. The theorems are extended to real meromorphic functions of the form $F(z)=A-B^{2} z+\left(r_{0} / z\right)+\sum_{1}^{\infty}\left\{\left[r_{j} /\left(z-c_{j}\right)\right]+m\left(r_{j} / c_{j}\right)\right\}$ with $m=0$ or 1 , and $A$ and $B$ real. (Received January 23,1948 .)

\section{O. W. Rechard: A note on the extension of rectangle functions.}

Let $\phi(R)$ be a real, finite, single-valued, non-negative function defined on the class $K$ of oriented closed rectangles $R$ contained in a fixed oriented closed rectangle $R_{0}$. A horizontal or vertical line segment $s$ with end points on the sides of $R_{0}$ is said to be regular in case for every rectangle $R$ in $K$ which is divided by $s$ into two rectangles $R_{1}$ and $R_{2}$ in $K$ it is true that $\phi(R)=\phi\left(R_{1}\right)+\phi\left(R_{2}\right)$. From an earlier result obtained by Rechard and Reichelderfer (Bull. Amer. Math. Soc. Abstract 53-5-224 accepted for publication in the Duke Math. J. vol. 15 (1948)) it follows that $\phi$ admits a completely additive extension to an additive class of subsets of $R_{\mathbf{0}}$, including all Borel sets in $R_{0}$, if and only if it satisfies the following conditions: (i) the class of regular lines in $R_{0}$ is everywhere dense both vertically and horizontally; (ii) if a descending sequence $R_{1}, R_{2}, \cdots$, of rectangles in $K$ has as a product the rectangle $R$ in $K$, then $\lim \phi\left(R_{n}\right)=\phi(R)$. A similar result is established for functions of oriented open rectangles. In this case, the sequence of rectangles in condition (ii) above is ascending with its sum equal to the rectangle $R$. (Received January 23, 1948.)

\section{Applied Math fmatics}

\section{M. Z. Krzywoblocki: On two-dimensional steady laminar jets in compressible viscous fluids.}

Bickley and Schlichting solved the equations of the velocity distribution in a steady two-dimensional laminar jet in incompressible fluid. In the present paper the problem of jets in compressible viscous fluids is solved. The following equations were taken into account: two equations of motion, continuity, state and energy. The two velocity components, the density and the temperature are given in the forms of the sequences of functions. (Received January 14, 1948.) 
221t. M. J. Norris: Coordinate transformations from the operational viewpoint.

It is shown that space-time coordinate transformation equations similar to the Lorentz equations can be obtained following the operational viewpoint of Bridgman. The problem treated is that in which the motions are restricted to one dimension. A local time for each observer is defined operationally, and then the time and distance of an observed event for a particular observer are defined operationally in terms of the observer's local time. The transformation equations are then derived. The methods are entirely elementary and are adequate to handle the case of non-uniform motion. (Received January 26,1948 .)

\section{GEOMETRY}

\section{P. M. Pepper: Local symmetry in metric spaces.}

Let $m$ be a point of a metric space $S, \delta$ a real number not less than 0 , and $S_{\delta}(m)$ the set of all points $s$ in $S$ for which $x m<\delta$. If $\Delta$ is the least upper bound of numbers $\delta \geqq 0$ with the property that if $x$ is in $S_{\delta}(m)$ then there exists a point $y_{x}$ in $S$ such that $x y_{x}=2 x m=2 m y_{x}$, then $S$ is said to have local symmetry about the center $m$ with the modulus $\Delta$. The modulus may be infinite in which case the word local is dropped. If $S$ is locally symmetric about two of its points $m_{0}, m_{1}\left(m_{0} \neq m_{1}\right)$ with respective moduli $\Delta_{0}, \Delta_{1}$, and if $m_{0} m_{1}<\max \left(\Delta_{0}, \Delta_{1}\right)$, then the diameter of $S$ is at least $\max \left[2 \min \left(\Delta_{0}, \Delta_{1}\right)\right.$ $\left.-2 m_{0} m_{1}, 2 m_{0} m_{1}\right]$. In case both moduli are infinite, the diameter of $S$ is infinite. A corollary of this theorem is: If $S$ is a complete, convex, metric space (of at least two points) which is locally symmetric about each of its points with moduli bounded away from 0 by a number $\Delta>0$, then the diameter of $S$ is not less than $2 \Delta$. The metric space $S$ is said to have local open-symmetry about its point $m$ with modulus $\Delta$ if and only if $\Delta$ is the least upper bound of numbers $\delta$ such that for each $\epsilon>0$ there exists a function $f_{\mathrm{e}}$ which maps the points $x$ of $S_{\delta}(m)$ into $S$ in such a way that $x f_{\epsilon}(x) \geqq 2 x m-\epsilon$ and $\left|f_{\epsilon}(x) m-x m\right| \leqq \epsilon$. The first theorem stated above for local symmetry holds also for local open-symmetry. (Received January 21, 1948.)

\section{Statistics and Probability}

\section{3t. Francis Regan and W. C. Kalinowski: A postulational treat-} ment of the probability for certain types of emissions.

The transmutation of radioactive substances is accompanied with the emission of certain types of rays. Since these emissions occur spontaneously and at random, the problem of radioactive transmutation can be treated statistically, that is, according to the laws of probability. This physical phenomenon is given a mathematical interpretation, in that a system of postulates is set up whereby the probability of the occurrence of $n$ points of a set $E$ (half open interval closed on the left) beginning at the instant $t$ may be obtained. Probabilities are also found for a finite number of nonabutting intervals. These results are then used to include a set of points $E$ whose frontier points are of measure zero. (Received January 21, 1948.)

\section{TOPOLOGY}

224. R. H. Bing: A convex metric for a locally connected continuum.

If $D(x, y)$ is a distance function, it is said to be a convex metric if for each pair of points $x, y$, there is a point $z$ such that $D(x, z)=D(z, y)=(1 / 2) D(x, y)$. If $M_{i}(i=1,2)$ 
is a compact continuum with a convex metric $D_{i}$ and $M_{1}$ intersects $M_{2}$, then there is a convex metric $D_{3}$ on $M_{1}+M_{2}$ that preserves $D_{1}$ on $M_{1}$. A necessary and sufficient condition that a compact $n$-dimensional metric space have a convex metric is that it be locally connected. (Received January 24, 1948.)

\section{S. B. Myers: Spaces of continuous functions.}

Let $B(X)$ be the Banach space of all real bounded continuous functions on a completely regular space $X$. A subspace $M$ of $B(X)$ is called completely regular over $X$ if given any closed set $K \subset X$ and any $x_{0} \in X-K$, there is a $b \in M$ such that $b\left(x_{0}\right)=\|b\|$ and, for $x \in K$, sup $|b(x)|<\|b\|$. The author has previously shown (Ann. of Math. vol. 49 (1948)) that if $B$ is a Banach space there is at most one compact $X$ such that $B$ is equivalent to a linear subspace of $B(X)$ completely regular over $X$. A further study is made here of the space $B(X)$ and its subspaces completely regular over $X$. For example, although $B(X)$ cannot be reflexive unless $X$ is finite, and $B(X)$ cannot be separable unless $X$ is compact metric, nevertheless (1) if $X$ is separable metric, there is a closed separable linear subspace of $B(X)$ which is completely regular over $X,(2)$ if $X$ is homeomorphically imbeddable in euclidean $n$-space, there is an $n+2$-dimensional linear subspace of $B(X)$ completely regular over $X$. (Received January 24, 1948.)

R. H. BRUCK, Associate Secretary 